\title{
The Allosteric Hemoglobin Effector ITPP Inhibits Metastatic Colon Cancer in Mice
}

Limani, Perparim ; Linecker, Michael ; Schneider, Marcel A ; Kron, Philipp ; Tschuor, Christoph ; Kachaylo, Ekaterina ; Ungethuem, Udo ; Nicolau, Claude ; Lehn, Jean-Marie ; Graf, Rolf ; Humar, Bostjan ; Clavien, Pierre-Alain

DOI: https://doi.org/10.1097/SLA.0000000000002431

Posted at the Zurich Open Repository and Archive, University of Zurich ZORA URL: https://doi.org/10.5167/uzh-139098

Journal Article

Published Version

Originally published at:

Limani, Perparim; Linecker, Michael; Schneider, Marcel A; Kron, Philipp; Tschuor, Christoph; Kachaylo, Ekaterina; Ungethuem, Udo; Nicolau, Claude; Lehn, Jean-Marie; Graf, Rolf; Humar, Bostjan; Clavien, Pierre-Alain (2017). The Allosteric Hemoglobin Effector ITPP Inhibits Metastatic Colon Cancer in Mice. Annals of Surgery, 266(5):746-753.

DOI: https://doi.org/10.1097/SLA.0000000000002431 


\title{
The Allosteric Hemoglobin Effector ITPP Inhibits Metastatic Colon Cancer in Mice
}

\author{
Perparim Limani, MD, ${ }^{*}$ Michael Linecker, MD, ${ }^{*}$ Marcel A. Schneider, MD, ${ }^{*}$ Philipp Kron, MD, ${ }^{*}$ \\ Christoph Tschuor, MD, ${ }^{*}$ Ekaterina Kachaylo, PhD, ${ }^{*}$ Udo Ungethuem, ${ }^{*}$ Claude Nicolau, PhD, $\dagger$ \\ Jean-Marie Lehn, PhD, $\ddagger$ Rolf Graf, PhD, ${ }^{*}$ Bostjan Humar, PhD, ${ }^{*}$ and Pierre-Alain Clavien, MD, PhD*
}

\begin{abstract}
Objective: To test the effects of enhanced intracellular oxygen contents on the metastatic potential of colon cancer.

Background: Colorectal cancer is the commonest gastrointestinal carcinoma. Distant metastases occur in half of patients and are responsible for most cancer-related deaths. Tumor hypoxia is central to the pathogenesis of metastases. Myo-Inositoltrispyrophosphate (ITPP), a nontoxic, antihypoxic compound, has recently shown significant benefits in experimental cancer, particularly when combined with standard chemotherapy. Whether ITPP protects from distant metastases in primary colon cancer is unknown.

Methods: ITPP alone or combined with FOLFOX was tested in a mouse model with cecal implantation of green fluorescent protein-labeled syngeneic colorectal cancer cells. Tumor development was monitored through longitudinal magnetic resonance imaging-based morphometric analysis and survival. Established serum markers of tumor spread were measured serially and circulating tumor cells were detected via fluorescence measurements.

Results: ITPP significantly reduced the occurrence of metastases as well as other indicators of tumor aggressiveness. Less circulating tumor cells along with reduction in malignant serum markers (osteopontin, Cxc112) were noted. The ITPP benefits also affected the primary cancer site. Importantly, animals treated with ITPP had a significant survival benefit compared with respective controls, while a combination of FOLFOX with ITPP conferred the maximum benefits, including dramatic improvements in survival (mean 86 vs $188 \mathrm{~d}$ ). Conclusions: Restoring oxygen in metastatic colon cancer through ITPP inhibits tumor spread and markedly improves animal survival; an effect that is enhanced through the application of subsequent chemotherapy. These promising novel findings call for a clinical trial on ITPP in patients with colorectal cancer, which is under way.
\end{abstract}

From the *Department of Surgery and Transplantation, Swiss Hepato-pancreatobiliary (HPB) and Transplantation Laboratory, University Hospital Zurich, Zurich, Switzerland; †Friedman School of Nutrition Science and Policy, Tufts University, Boston, MA; and $\ddagger$ Institute of Supramolecular Science and Engineering, University of Strasbourg, Strasbourg, France.

$\mathrm{BH}$ and $\mathrm{P}-\mathrm{AC}$ shared senior authorship.

This study was supported by the Klinischer Forschungsschwerpunkt of the University Hospital Zurich, the Sassella Foundation (Nr. 12/05 and 13/01), the Candoc Forschungskredit (University of Zurich, FK-13-030), the Liver and Gastrointestinal Disease and the Yvonne Jacob Foundations, and the Swiss Cancer League (KFS-3262-08-2013).

The authors of this manuscript contributed to the study design, analysis, interpretation of data, drafting the article, revising it, and final approval of the version to be published. The authors confirm that there is no one else who fulfills the criteria but has not been included as an author.

The authors report no conflicts of interest.

Supplemental digital content is available for this article. Direct URL citations appear in the printed text and are provided in the HTML and PDF versions of this article on the journal's Web site (www.annalsofsurgery.com).

Reprints: Pierre-Alain Clavien, MD, PhD, Department of Surgery and Transplantation, University Hospital Zurich, Raemistrasse 100, CH-8091 Zürich, Switzerland. E-mail: clavien@access.uzh.ch.

Copyright (C) 2017 Wolters Kluwer Health, Inc. All rights reserved.

ISSN: 0003-4932/16/XXXX-0001

DOI: $10.1097 /$ SLA.0000000000002431
Keywords: colorectal cancer, hypoxia, ITPP, metastases, myoinositoltrispyrophosphate, tumor angiogenesis

(Ann Surg 2017;xx:xxx-xxx)

olorectal cancer (CRC) is the third most common malignant disease. ${ }^{1,2}$ About half of patients eventually present with distant metastases, greatly contributing to the cancer-related death toll. ${ }^{3}$ Besides improving the treatment of metastases, death rates might be lowered with better strategies against locally invasive CRC that has not spread to distant sites yet. Stage III disease currently is being treated with surgery and adjuvant chemotherapy (folinic acid, fluorouracil, oxaliplatin [FOLFOX] and similar regimens). A significant proportion of stage III patients however do not seem to benefit from chemotherapy. ${ }^{4}$

Over the last decade, novel biological agents with antiangiogenic activity (eg, bevacizumab) have been added to standard chemotherapy against metastatic CRC. Despite early successes, ${ }^{5-7}$ antiangiogenic approaches seem to be less effective than initially anticipated and do not confer any benefits in stage III CRC., ${ }^{4}$

Rapid tumor growth leads to a paucity of vessels in the core, causing an insufficient supply with oxygen and nutrients. The developing hypoxia provokes a tumor response that is driven through the hypoxia-inducible factors (HIFs) and includes vascular endothelial growth factor (VEGF)-mediated angiogenesis. ${ }^{9}$ Antiangiogenic therapy has been designed to starve tumors by pruning vessels. ${ }^{5}$ However, the lack of vessels may increase hypoxia and thereby foster the hypoxic response, a recognized promoter of malignant conversion. ${ }^{8}$ Accordingly, anti-VEGF treatment for CRC metastasis overall comes with little benefit in terms of survival, and may even worsen outcomes in other cancers. ${ }^{8,10}$

Since tumor hypoxia is a key driver of malignancy, targeting hypoxia directly may be more effective against dissemination than antiangiogenic approaches. ${ }^{1-13}$ Myo-inositoltrispyrophosphate (ITPP) is a synthetic derivative of myoinositol hexakisphosphate, an allosteric effector of hemoglobin. ${ }^{14}$ Like the latter, ITPP decreases the binding affinity of hemoglobin to oxygen, leading to an enhanced release of oxygen specifically under hypoxic conditions. Additionally, ITPP readily accumulates in erythrocytes, enabling an increased oxygen release also in vivo. ${ }^{15}$ Thus, ITPP is able to elevate oxygen pressure in hypoxic tumors, thereby suppressing the HIFs and the hypoxic tumor response, including the angiogenic switch. ${ }^{16}$ Its favorable toxicity profile animated the assessment of ITPP in several animal cancer models. Importantly, ITPP treatment improves survival of cancer-bearing animals, particularly if combined with cytotoxic agents. ${ }^{16-23}$ Recently, we observed a prolonged survival through ITPP in a mouse model of established CRC liver metastasis, with a marked potentiation of efficacy when combined with FOLFOX.${ }^{16,24}$ However, whether ITPP can counteract the spread of CRC to distant sites is unknown. Likewise, whether an antimetastatic effect would translate into an improved survival has not been addressed so far. 
Here, we used a syngeneic orthotopic CRC model based on cecal implantation of CRC cells to determine whether ITPP has the potential to inhibit metastatic spread leading to a prolonged survival, and whether a combination with standard chemotherapy might potentiate resulting benefits.

\section{METHODS}

\section{Animals}

C57B1/6 mice (Harlan, Itlingen, Switzerland) aged 10 to 12 weeks were kept on a 12-hour day/night cycle with free access to food and water. All animal experiments were in accordance with Swiss federal animal regulations and approved by the cantonal veterinary office of Zurich.

\section{Cell Culture, Animal Procedures, Treatment, and Small Animal MRI}

Green fluorescent protein (GFP)-labeled MC-38 CRC cells (C57B1/6-syngeneic) were cultured under standard conditions. ${ }^{24}$ Cecal injection of tumor cells was performed as reported. ${ }^{25}$ ITPP was synthetized and administered as a solution containing calcium chloride to balance its chelating effects as described. ${ }^{15,16}$ The procedures for FOLFOX treatment ${ }^{16,26}$ and the small animal magnetic resonance imaging protocol have been reported. ${ }^{24}$ The treatment schedule including experimental groups is depicted in Figure 1.

\section{Histological Examination, Immunohistochemistry, GFP detection}

Hematoxylin and Eosin staining and immunohistochemistry for KI67 was performed on 3- $\mu \mathrm{m}$ archived sections as reported. ${ }^{16}$ KI67 positivity was digitally assessed using AnalySIS ${ }^{\mathrm{D}} 5.0$ software (Olympus, Hamburg, Germany). The numbers of circulatory tumor cells were indirectly determined by measuring serum GFP-fluorescence using a Biotek Cytation 3 Reader (Biotek, Luzern, Switzerland).

\section{Quantitative Real-time Polymerase Chain Reaction}

Total RNA was extracted from $50 \mathrm{mg}$ tissue using Trizol reagent (Invitrogen, Basel, CH, Switzerland). Quantitative real-time PCR was performed on cDNA (ThermoScript reverse-transcription PCR System, Invitrogen) using an ABI Prism 7500 Sequence Detector System and predesigned assays (PE Applied Biosystems, Rotkreuz, $\mathrm{CH}$, Switzerland): Cdh1, Mm01247357_m1; Hifla, Mm00468869_m1; Hif2a, Mm01236112_m1; Ocln, Mm00500912_m1; Snail, Mm00441533_g1; Src, Mm00436785_m1, Twist1, Mm00442036_ m1; Vegfa, Mm01281449_m1; Vim, Mm01333430_m1. Results represent mean fold induction $\left(2^{-\Delta \mathrm{Ct}}\right) \pm \mathrm{SD}$ relative to the normalization control reagent 18 SrRNA.
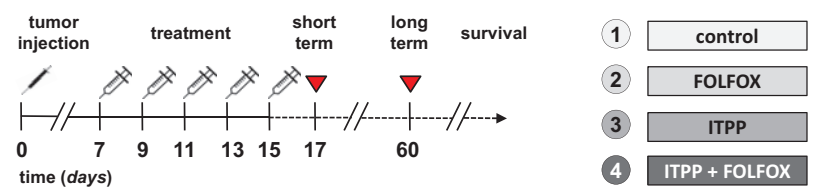

FIGURE 1. Experimental setup for the study of anticancer treatments in a syngeneic, orthotopic mouse model of invasive CRC. The cecal injection of MC-38 cells leads to MRI-detectable, intestinal tumors at day 7. Treatment (with ITPP, FOLFOX, or a combination thereof) was initiated at day 7 and repeated 4 times every other day. Mice were analyzed for short-term effects at day 17 and for long-term effects at day 60 (6 weeks after treatment).

\section{Enzyme-linked Immunosorbent Assay (ELISA)}

ELISAs for VEGF (order number DY493), osteopontin (MOST00), CXCL12 (MCX120) were from R\&D Systems (Minneapolis, MN); for HMGB1 (ST51011) from IBL International (Hamburg, D); and for HIF1A/HIF2A (E90798Mu/E93466Mu) from NewLife BioChemEX (Bethesda, MD). Triplicate measurements were performed.

\section{Statistical Analysis}

GraphPad Prism (GraphPad Software Inc, La Jolla, CA) was used. Groups were compared with a $t$ test or 1-way analysis of variance with post-hoc Bonferroni correction where appropriate. Significances were categorized according to levels ( $n s$ nonsignificant, $\left.{ }^{*} P<0.05,{ }^{* *} P<0.01,{ }^{* * *} P<0.001\right)$. Mean \pm SD is shown. Six animals/group were analyzed unless otherwise stated.

\section{RESULTS}

\section{ITPP Downregulates HIF Activities in the Primary Tumor}

Hypoxia leads to the activation of the HIFs, chief transmitters of the hypoxic tumor response. Consistent with previous findings, ${ }^{16}$ ITPP treatment of mice (Fig. 1) reduced both Hifla/2a gene expression and HIF1A/2A protein levels in the primary CRC (Fig. 2A). Importantly, reductions were seen immediately after treatment but also 6 weeks later, indicating a lasting ITPP effect. The downregulation of nuclear HIF1A/2A was associated with reduced tumoral expression of angiogenic Vegfa, a key transcriptional target of the HIFs (Fig. 2B). Accordingly, circulating VEGF protein was lowered in sera following ITPP treatment. Therefore, ITPP treatment leads to a stable inhibition of HIF activities in the primary tumor.

\section{ITPP Slows the Growth and the Malignant Potential of the Primary Tumor}

The inhibition of HIF activities through ITPP should slow tumor development. ${ }^{16}$ When assessing the volume of the primary CRC, ITPP treatment reduced the size both in a short and long term (Fig. 3A), along with congruent changes in the proliferation marker KI67 (Fig. 3B).

HIFs are known promoters of the epithelial-to-mesenchymal transition (EMT), a key step in the acquisition of malignant behavior, where epithelial tumor cells gain mesenchymal traits that enable them to invade and migrate to distant sites. ${ }^{27}$ We measured in tumors a panel of EMT inducers (Twist, Snail, Src), and mesenchymal (Vim) as well as epithelial $(O c l n, C d h 1)$ markers. The ITPP-induced changes (upregulation of epithelial markers at the expense of EMT inducers and mesenchymal markers, Fig. 3C) were fully consistent with a lasting suppression of the EMT.

In agreement, serum markers of systemic malignancy (osteopontin, CXCL12) and inflammation (HMGB1) fostered through hypoxia $^{28}$ were diminished by ITPP in a short and long term (Fig. 3D). Importantly, ITPP treatment also reduced serum GFP signals, an indirect measure for the number of circulating, GFPtagged tumor cells (Fig. 3E).

Together, these findings indicate that ITPP treatment not only slows the growth of the primary, but also reduces its malignant potential in a lasting way.

\section{ITPP Counteracts the Development of Hepatic CRC Metastases and Displays Additive Effects With Chemotherapy}

To determine whether the ITPP-induced switch to a more benign tumor phenotype translates into an effective inhibition of metastatic spread, we determined the frequency and number of 

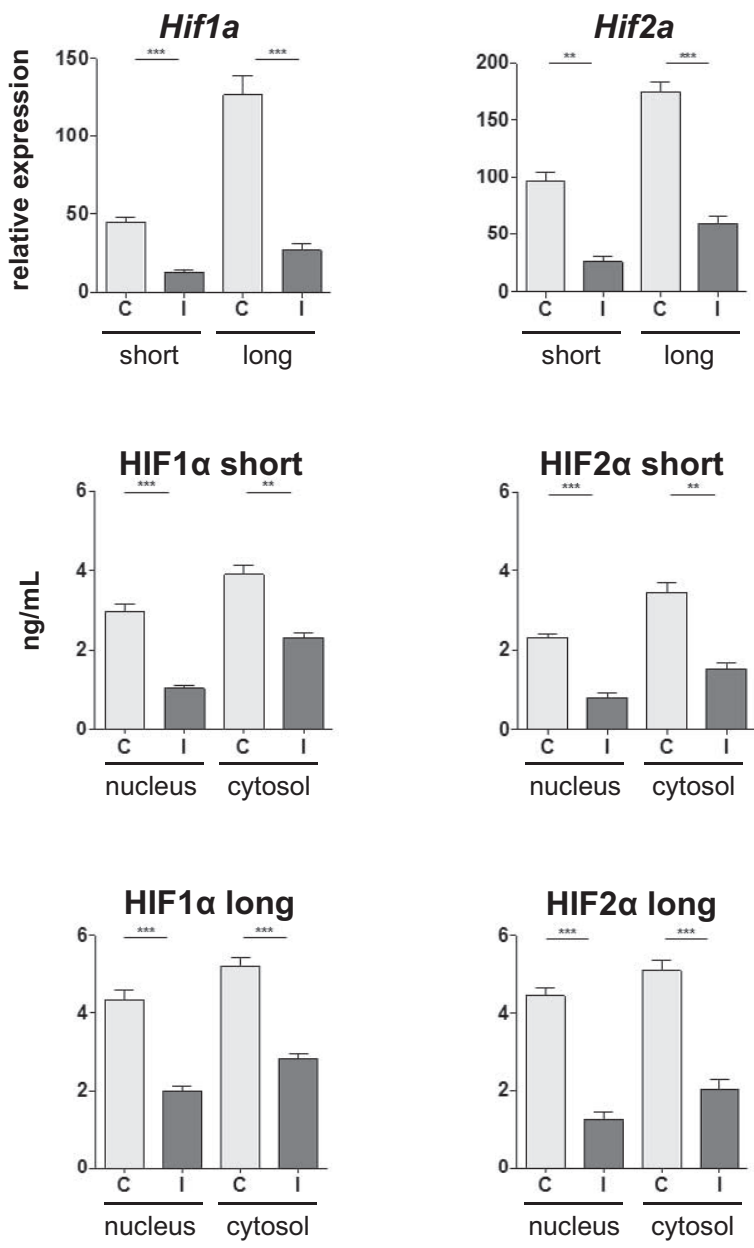

A
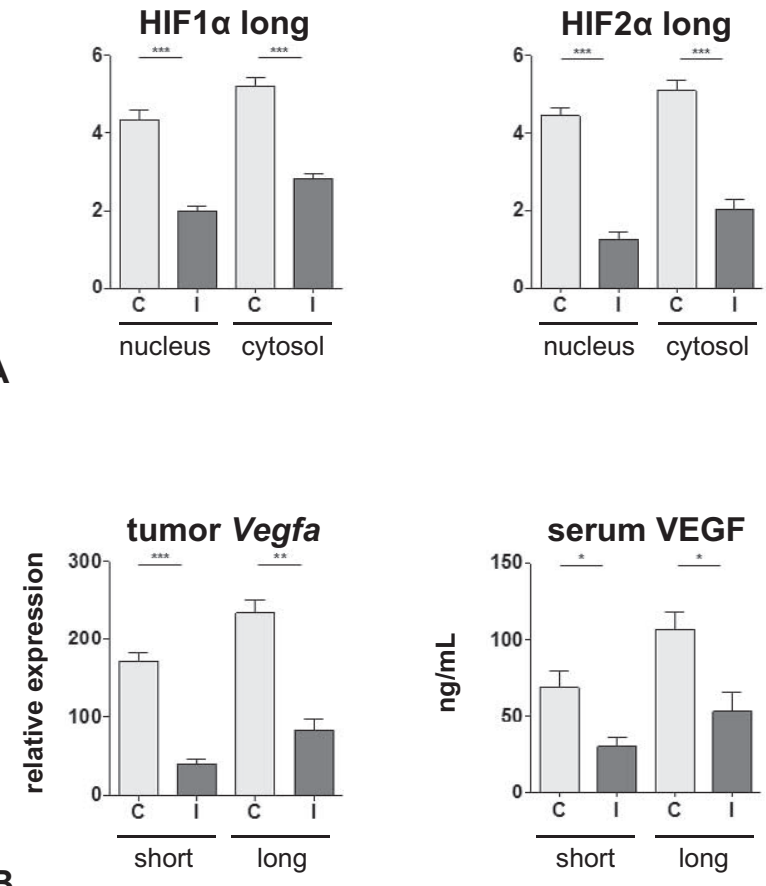

FIGURE 2. ITPP effects on HIF activities in the primary tumors. A, Hifl a/2 $a$ gene and HIF1A/2A protein expression in control (C) and ITPP-exposed (I) tumors immediately (short) or 6 weeks (long) after treatment. Note the reductions in nuclear HIFs. B, Tumoral gene expression for the HIF transcriptional target Vegfa and serum VEGF protein levels. For all figures: $n=6 /$ group unless otherwise stated; ${ }^{*} P<0.05,{ }^{* *} P<0.01,{ }^{* * *} P<0.001$.

metastases in the liver, the preferred site for CRC spread. To better appreciate the clinical efficacy, we compared ITPP effects with those of FOLFOX, the standard treatment for invasive CRC.
In a first step, we assessed the FOLFOX effects on the volume of the primary so as to confirm its anticancer activity in our model. Given that ITPP can enhance the effects of conventional chemotherapy, ${ }^{16,20}$ we included an animal group exposed to combined ITPPFOLFOX treatment. FOLFOX monotherapy reduced primary tumor volume both in a short and long term with effects akin to those of ITPP (Fig. 4A). Interestingly, the ITPP-FOLFOX combination doubled the volume reductions achieved through each monotherapy alone, suggesting ITPP has an additive impact on primary FOLFOX effects (Fig. 4A). Therefore, FOLFOX is active in our model, while the combination of ITPP-FOLFOX induces a primary tumor response superior over monotherapy.

In a second step, we assessed the number of animals with liver metastasis and the number of colonies per animal at 6 weeks after treatment. In untreated controls, $\sim 60 \%$ of animals presented with liver metastasis. This number was reduced to $\sim 50 \%$ with FOLFOX, $\sim 30 \%$ with ITPP, and 25\% with ITPP-FOLFOX (Fig. 4B, see also Supplementary Fig. S1, http://links.lww.com/SLA/B298). Likewise, the number of metastases per animal was reduced through the treatment from $\sim 18$ in untreated controls to about 16,10 , and 7 in the FOLFOX, ITPP, and ITPP-FOLFOX groups, respectively (Fig. 4C). These observations suggest a stronger antimetastatic effect for ITPP than for FOLFOX; however, the combination of the 2 treatments led to the most potent suppression of metastatic spread to the liver.

\section{ITPP Extends Survival of Mice With Metastasizing CRC and Potentiates the Survival Benefits of Chemotherapy}

The most relevant endpoint with regards to cancer treatment is survival. To assess whether the effects of ITPP on primary/secondary CRC and on chemotherapy efficacy impact on survival of tumorbearing mice, we repeated the above experiments for a KaplanMeier analysis (Fig. 5).

The mean survival for tumor-bearing mice without treatment was 75 days from treatment end. Survival was extended to about 111 days ( $+48 \%$ relative to controls) through both ITPP and FOLFOX monotherapies. Intriguingly, the combination of ITPP and FOLFOX prolonged survival to 170 days $(+127 \%)$. Therefore, the combination of ITPP and FOLFOX has a stronger effect on survival than on tumor load, suggesting ITPP has some synergistic effect with FOLFOX on life expectancy in our model. We conclude that ITPP has a major potential in improving the outcome of invasive CRC when combined with standard chemotherapy.

\section{DISCUSSION}

The most relevant cause of cancer-related death is the metastatic spread of disease. ${ }^{1-4}$ A reduction of malignant potential before the dissemination to distant sites might hence improve survival. Cytotoxic chemotherapies such as FOLFOX regimen are currently the treatment of choice for stage III CRC; however, the resulting survival benefits are small. ${ }^{4,29}$ The need for novel approaches in the management of stage III disease remains. Agents that reduce the risk of developing metastases and that can be integrated into the current standard of care are particularly desirable. In this study, we demonstrate that the nontoxic, antihypoxic compound ITPP (i) inhibits the growth and malignant potential of experimental stage III CRC, (ii) is more efficacious than FOLFOX in reducing the progression of $\mathrm{CRC}$ to the liver, and (iii) markedly enhances the FOLFOX effects on primary tumor growth, metastatic development, and survival. Therefore, ITPP meets the key requirements to improve the outcomes of standard chemotherapy for invasive CRC in experimental settings. 
tumor volume

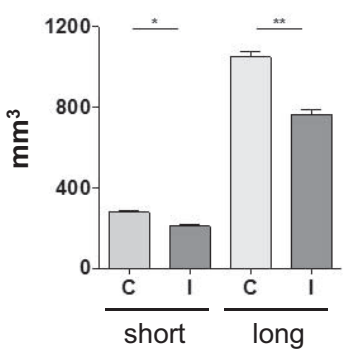

KI67

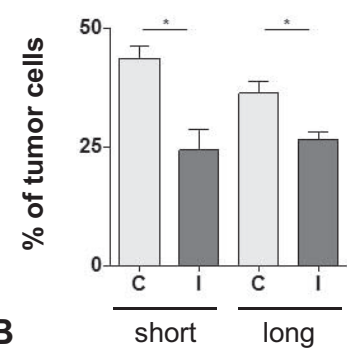

EMT short

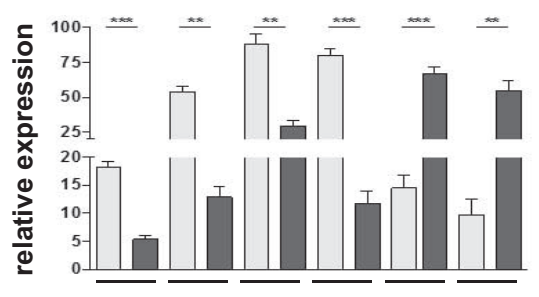

C

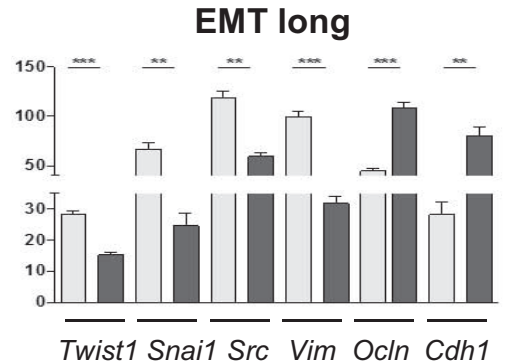

Twist1 Snai1 Src Vim Ocln Cdh1
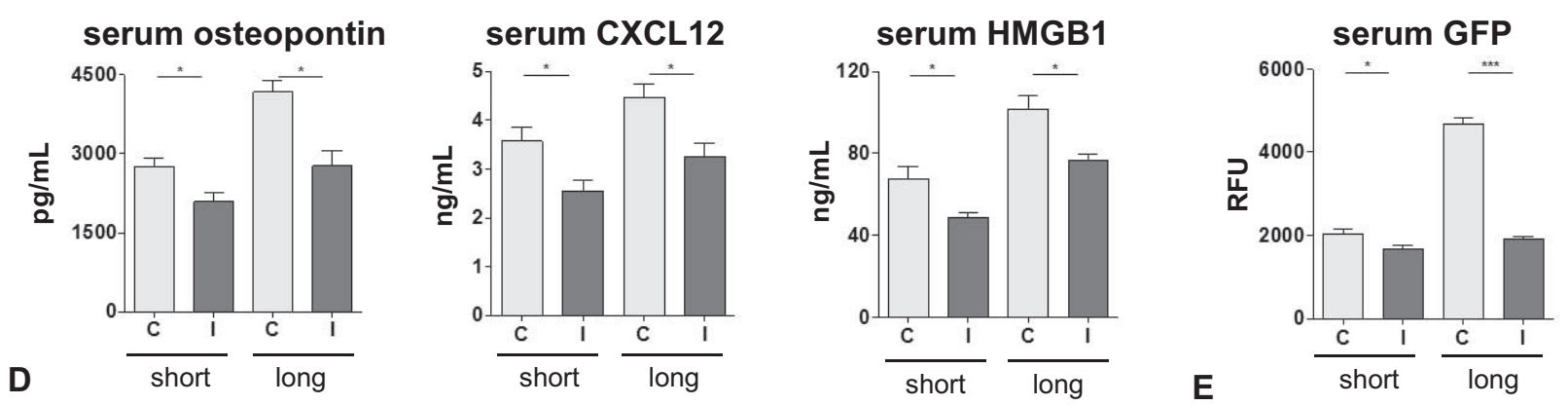

FIGURE 3. ITPP effects on growth and malignant potential of the primary. A, MRI-assessed tumor volume in control (C) and ITPPexposed (I) animals immediately (short) and 6 weeks (long) after treatment. B, Corresponding KI67-proliferation indices. C, Tumoral gene expression for EMT-associated markers immediately and 6 weeks after treatment. Dark bars correspond to ITPP-treated animals. D, Serum osteopontin, CXCL12, and HMGB1 protein levels. E, Serum GFP fluorescence as a measure for circulating tumor cells.

ITPP efficiently inhibited HIF activities in the primary CRC. The HIFs are key mediators of the hypoxic tumor response, and we have previously shown in established liver metastases (based on a similar syngeneic model) that ITPP-mediated tumor oxygenation and associated HIF inhibition leads to a global suppression of the hypoxic response, including the angiogenic switch, inhibition of the Warburg effect (ie, a shift from glucose usage to $\beta$-oxidation), improved immune infiltration, the skewing toward anti-cancerous macrophage action, and a suppression of malignant potential along with the promotion of an epithelial, differentiated tumor cell phenotype. ${ }^{16}$ Indeed, forced activation of the HIFs was sufficient to abort the beneficial effects of ITPP,${ }^{16}$ indicating that the working mode of ITPP fully depends on the inhibition of HIFs and the hypoxic response.

Here, we focused on the malignant potential, because of its direct relevance for metastatic spread. ITPP inhibited the hypoxiadriven EMT, ${ }^{27}$ consistent with its reported ability to promote in colon cancer the expression of CDX2, a master promoter of a differentiated intestinal phenotype. ${ }^{18}$ Moreover, hypoxia-associated inflammation is known to induce systemic promoters of malignancy (osteopontin, CXCL12), ${ }^{28}$ and ITPP significantly reduced their serum levels, including those of the key inflammatory mediator HMGB1. ITPP thus counteracts the epithelial degeneration that confers invasiveness to cancer cells, and reduces the promotion of their systemic spread, as evinced by the lesser numbers in circulating tumor cells. Given the current lack of direct evidence, however, it was important to demonstrate that ITPP does have an impact on the development of metastases at distant sites.

As expected from the phenotypic changes observed in the primary, ITPP was more efficient than FOLFOX in trimming the metastatic load. On the other hand, the survival benefit was similar for the 2 monotherapies. Survival strongly correlated with the longterm reductions in primary volume $\left(\mathrm{R}^{2}=-0.996\right)$, but also with the 
tumor volume

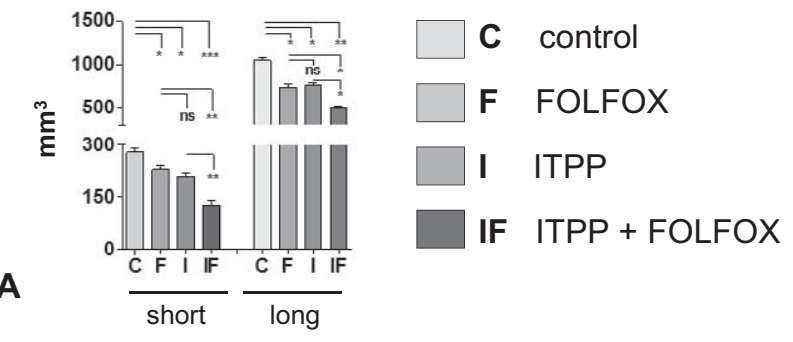

B
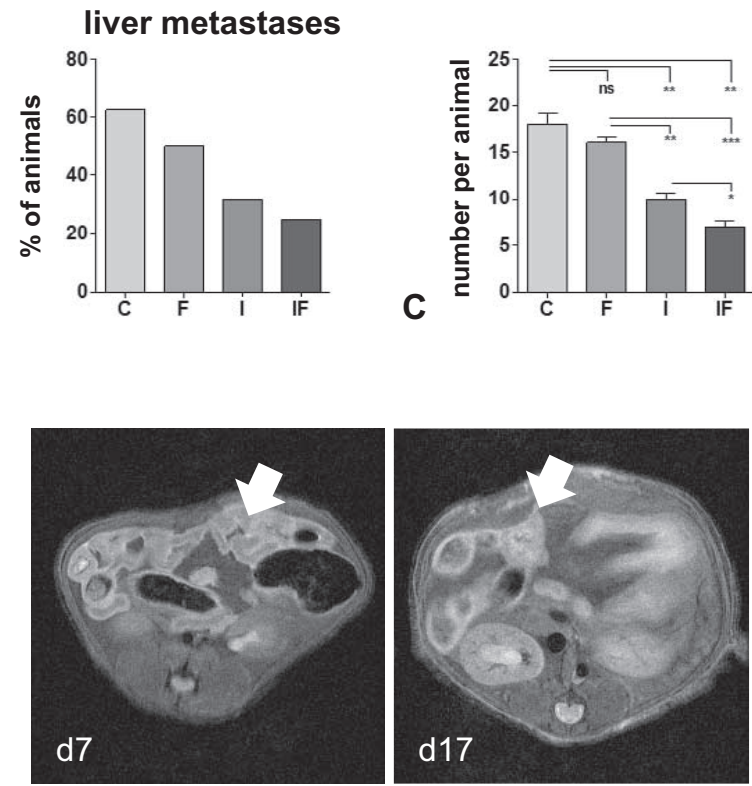

D
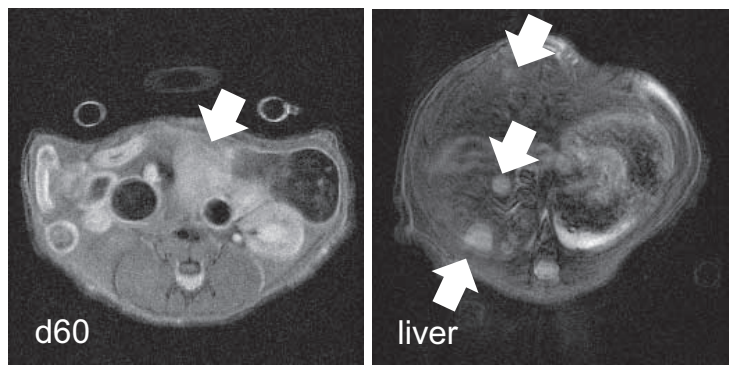

FIGURE 4. ITPP and FOLFOX effects on primary tumors and hepatic metastases. A, MRI-assessed primary tumor volume immediately and 6 weeks after the various treatments. B, Percentage incidence of liver metastases 6 weeks after treatments. C, Number of metastatic liver lesions per animal at 6 weeks after treatments. D, MRI scans showing examples of primary tumor development (d7, d17, d60) and metastatic lesions (liver) at 6 weeks after treatment.

metastatic load (ie, incidence multiplied by metastatic numbers, $\mathrm{R}^{2}=-0.896$ ), suggesting that both the primary and secondary tumors have contributed to the animals' death. As such, our model may not fully reflect human $\mathrm{CRC}$, where metastasis remains the main cause of death. Furthermore, our findings are based on one model only. Given that hypoxia is a key driver of malignant conversion, ${ }^{27}$ it however is plausible that targeting hypoxia through ITPP will generally reduce metastatic risks.

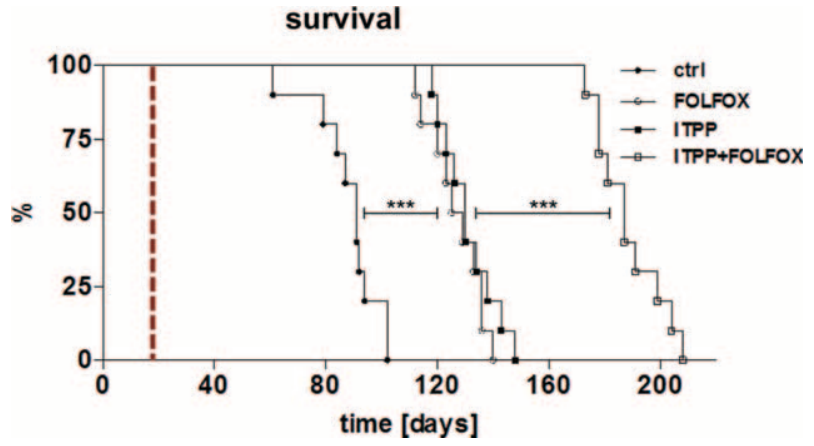

FIGURE 5. Survival of tumor-bearing mice. Survival count in days starts with day 17 (marked by a dashed line), that is, immediately after treatment. $\mathrm{n}=8-10 /$ group.

The strongest effect on metastatic load and survival was achieved through the ITPP-FOLFOX combination treatment. ITPP monotherapy is unlikely sufficient to advance the management of invasive CRC, but 1 reported key effect of ITPP is the normalization of tumor-associated vasculature. ${ }^{16,20}$ Tumor-induced vessels often are dysfunctional and leaky, leading to irregular supply of oxygen, nutrients, but also medications. ${ }^{30}$ We have shown that ITPP causes stable normalization of tumor vasculature ${ }^{16}$; vessel normalization, in turn, improves the delivery of cytotoxics to cancer cells, explaining the synergistic effects of ITPP plus FOLFOX on survival. The lasting normalization effects point to another key advantage of ITPP, that is, the provision of a therapeutic window that enables the sequential use of ITPP followed by standard chemotherapy. ${ }^{16}$ In this way, potential drug-drug interactions or cumulative toxicities should be minimized, enabling the use of ITPP without compromising the standard of care.

The ability of ITPP to normalize vessels further implies that the compound will improve delivery of any anticancer agent and hence may be applied for a wide variety of diseases. In support of the latter, ITPP has been shown to markedly improve the efficacy of gemcitabine in experimental pancreatic cancer. ${ }^{20}$ Moreover, ITPP does not affect cancer cells directly, ${ }^{16}$ but acts on the tumor environment (eg, hemoglobin). ${ }^{15}$ Unlike cancer cells, the environment should be genetically stable, implying that the development of resistance against ITPP is an unlikely event. Genetic instability however may affect ITPP efficacy; unstable cancer cells are likely to adapt to unfavorable conditions over time, producing malignant clones that thrive under hypoxia. It therefore is possible that the antihypoxic properties of ITPP will have little effect in very advanced stages. Only clinical testing will reveal the indications amenable to ITPP. Importantly, ITPP has not been associated with any toxicity either in animals (also at doses well above those used here) ${ }^{16}$ or in healthy volunteers (http://normoxys.com/clinical-trial-results/). We thus have initiated a first-in-patient phase $\mathrm{Ib} / \mathrm{II} a$ trial to assess the safety of ITPP and to obtain a first estimate for the ITPP effects on standard-of-care treatment. ${ }^{31}$

In summary, our findings indicate a potential for ITPP in improving the existing management of invasive CRC. ITPP's most important feature is its ability to enhance the efficacy of standard chemotherapy against primary and secondary tumors. Thus, ITPP can be integrated into current treatment approaches without compromising standard of care. Moreover, ITPP alone has antimetastatic activity besides its effects on the primary. Together with its low toxicity ${ }^{16}$ and its beneficial effects on physical capacity, ${ }^{32}$ the use of ITPP in the clinic deserves promotion. 


\section{ACKNOWLEDGMENTS}

The authors thank Eleonora Maurizio, Pia Fuchs, and Christine Lohmann for excellent technical assistance.

\section{REFERENCES}

1. Van Cutsem E, Cervantes A, Adam R, et al. ESMO consensus guidelines for the management of patients with metastatic colorectal cancer. Ann Oncol. 2016;27:1386-1422.

2. Ferlay J, Soerjomataram I, Dikshit R, et al. Cancer incidence and mortality worldwide: sources, methods and major patterns in GLOBOCAN 2012. Int J Cancer. 2015;136:E359-E386.

3. La Vecchia C, Bosetti C, Lucchini F, et al. Cancer mortality in Europe, 20002004, and an overview of trends since 1975. Ann Oncol. 2010;21:1323-1360.

4. Brenner H, Kloor M, Pox CP. Colorectal cancer. Lancet. 2014;383: 1490-1502

5. Hurwitz H, Fehrenbacher L, Novotny W, et al. Bevacizumab plus irinotecan, fluorouracil, and leucovorin for metastatic colorectal cancer. $N$ Engl J Med. 2004;350:2335-2342.

6. Wilhelm SM, Dumas J, Adnane L, et al. Regorafenib (BAY 73-4506): a new oral multikinase inhibitor of angiogenic, stromal and oncogenic receptor tyrosine kinases with potent preclinical antitumor activity. Int $J$ Cancer. 2011;129:245-255.

7. Chu QS. Aflibercept (AVE0005): an alternative strategy for inhibiting tumour angiogenesis by vascular endothelial growth factors. Expert Opin Biol Ther 2009;9:263-271.

8. Jain RK, Duda DG, Clark JW, et al. Lessons from phase III clinical trials on anti-VEGF therapy for cancer. Nat Clin Pract Oncol. 2006;3:24-40.

9. Pugh CW, Ratcliffe PJ. Regulation of angiogenesis by hypoxia: role of the HIF system. Nat Med. 2003;9:677-684.

10. Xu H, Rahimpour S, Nesvick CL, et al. Activation of hypoxia signaling induces phenotypic transformation of glioma cells: implications for bevacizumab antiangiogenic therapy. Oncotarget. 2015;6:11882-11893.

11. Paolicchi E, Gemignani F, Krstic-Demonacos M, et al. Targeting hypoxic response for cancer therapy. Oncotarget. 2016;7:13464-13478.

12. Hanahan D, Weinberg RA. The hallmarks of cancer. Cell. 2000;100:57-70.

13. Hanahan D, Weinberg RA. Hallmarks of cancer: the next generation. Cell. 2011;144:646-674.

14. Koumbis AE, Duarte CD, Nicolau C, et al. Tetrakisphosphates and bispyrophosphates of myo-inositol derivatives as allosteric effectors of human hemoglobin: synthesis, molecular recognition, and oxygen release. Chem Med Chem. 2011;6:169-180.

15. Fylaktakidou KC, Lehn JM, Greferath R, et al. Inositol tripyrophosphate: a new membrane permeant allosteric effector of haemoglobin. Bioorg Med Chem Lett. 2005;15:1605-1608.

16. Limani P, Linecker M, Kachaylo E, et al. Antihypoxic potentiation of standard therapy for experimental colorectal liver metastasis through myo-inositol trispyrophosphate. Clin Cancer Res. 2016;22:5887-5897.

17. Aprahamian M, Bour G, Akladios CY, et al. Myo-InositolTrisPyroPhosphate treatment leads to HIF-1alpha suppression and eradication of early hepatoma tumors in rats. Chembiochem. 2011;12:777-783.

18. Derbal-Wolfrom L, Pencreach E, Saandi T, et al. Increasing the oxygen load by treatment with myo-inositol trispyrophosphate reduces growth of colon cance and modulates the intestine homeobox gene Cdx2. Oncogene. 2013;32: 4313-4318.

19. Kieda C, El Hafny-Rahbi B, Collet G, et al. Stable tumor vessel normalization with $\mathrm{pO}(2)$ increase and endothelial PTEN activation by inositol trispyrophosphate brings novel tumor treatment. J Mol Med (Berl). 2013;91:883-899.

20. Raykov Z, Grekova SP, Bour G, et al. Myo-inositol trispyrophosphatemediated hypoxia reversion controls pancreatic cancer in rodents and enhances gemcitabine efficacy. Int J Cancer. 2014;134:2572-2582.

21. Sihn G, Walter T, Klein JC, et al. Anti-angiogenic properties of myo-inositol trispyrophosphate in ovo and growth reduction of implanted glioma. FEBS Lett. 2007;581:962-966.

22. Fornvik K, Zolfaghari S, Salford LG, et al. ITPP treatment of RG2 glioblastoma in a rat model. Anticancer Res. 2016;36:5751-5755.

23. Ignat M, Akladios CY, Lindner V, et al. Development of a methodology for in vivo follow-up of hepatocellular carcinoma in hepatocyte specific Trim24-null mice treated with myo-inositol trispyrophosphate. J Exp Clin Cancer Res. 2016;35:155.

24. Limani P, Borgeaud N, Linecker M, et al. Selective portal vein injection for the design of syngeneic models of liver malignancy. Am J Physiol Gastrointest Liver Physiol. 2016;310:G682-G688.
25. Tan $\mathrm{MH}$, Holyoke ED, Goldrosen MH. Murine colon adenocarcinoma: syngeneic orthotopic transplantation and subsequent hepatic metastases. J Natl Cancer Inst. 1977;59:1537-1544.

26. Robinson SM, Mann J, Vasilaki A, et al. Pathogenesis of FOLFOX induced sinusoidal obstruction syndrome in a murine chemotherapy model. J Hepatol. 2013;59:318-326.

27. Jiang J, Tang YL, Liang XH. EMT: a new vision of hypoxia promoting cancer progression. Cancer Biol Ther. 2011;11:714-723.

28. Ebos JM, Lee CR, Christensen JG, et al. Multiple circulating proangiogenic factors induced by sunitinib malate are tumor-independent and correlate with antitumor efficacy. Proc Natl Acad Sci U S A. 2007;104:17069-17074.

29. Adjuvant chemotherapy for localised colon cancer. Fluorouracil + folinic acid for node-positive, non-metastatic disease. Prescrire Int. 2011;20:46-49.

30. Multhoff G, Radons J, Vaupel P. Critical role of aberrant angiogenesis in the development of tumor hypoxia and associated radioresistance. Cancers (Basel). 2014;6:813-828

31. Limani P, Linecker M, Kron P, et al. Development of OXY111A, a novel hypoxia-modifier as a potential antitumor agent in patients with hepatopancreato-biliary neoplasms-protocol of a first Ib/IIa clinical trial. BMC Cancer. 2016;16:812.

32. Biolo A, Greferath R, Siwik DA, et al. Enhanced exercise capacity in mice with severe heart failure treated with an allosteric effector of hemoglobin, myo-inositol trispyrophosphate. Proc Natl Acad Sci U S A. 2009;106: 1926-1929.

\section{DISCUSSANTS}

\section{Peter A. Lodge (Leeds, UK):}

There is no doubt that this is an important and well carried out study and I congratulate the Zurich team for this excellent work. Thank you for allowing me to see the manuscript before the presentation and for altering the manuscript title to emphasise that this was a study of the effects of ITPP, rather than oxygen.

Your data suggest that the inhibition of hypoxia through increased release of oxygen from hemoglobin is the key mechanism underlying ITPP action and this inhibits tumor hypoxia, which in turn leads to HIF (hypoxia inducible factors) inactivation and the suppression of the hypoxic response. First, if this is true, this directly challenges the use of drugs that act against VEGF such as Bevacizumab. Are you able to comment further on that? Second, a significant proportion of patients will not benefit from combination chemotherapy. Can you speculate how efficacious (or how consistently effective) we should expect ITPP to be? Third, ITPP has been used in race horses and greyhounds to increase exercise capacity and I understand trials for heart failure are ongoing. It seems to be a new "wonder drug" - Are you taking it and should we all be?

\section{Response from Perparim Limani (Zurich, Switzerland):}

Thank you Professor Lodge for your appreciation of our paper and your highly relevant questions. Indeed, our data challenge the concept of anti-VEGF treatment (eg, Bevacizumab). In our previous work on experimental stage IV CRC (PMID: 27489288), we directly compared ITPP plus FOLFOX with anti-VEGF plus FOLFOX and experimentally documented the marked superiority of the ITPPFOLFOX combination in terms of survival benefits. The likely reason is that ITPP - by increasing oxygenation - inhibits the complete hypoxic tumor response (a recognized driver of malignancy), while anti-VEGF only counteracts the angiogenic response which possibly even worsens hypoxia. The latter might explain why anti-VEGF therapy is not consistently associated with an improved overall survival (eg, PMID: 27866194, 27840192, 27081084).

How efficacious ITPP will be in enhancing chemotherapy in patients remains to be assessed. We are running a clinical trial to evaluate ITPP in patients with hepato-pancreato-biliary neoplasms 
(ClincialTrials.gov ID: NCT02528526), which will give first data on the combination of ITPP with standard cytostatics. We believe ITPP will be efficient in situations, where the tumor still responds to oxygenation changes. If disease, however, is too progressed (ie, after years of chronic hypoxia), cancer cells might not care anymore about oxygen levels and may become less sensitive toward ITPP.

You obviously did good research on the topic. ITPP indeed seems to be used as a doping agent, largely due to its performanceenhancing capacities (increased oxygen availability during exercising). Currently, ITPP is administered via infusion (no oral formulations exist as yet), therefore inappropriate for private use. It has some potential for a wonder drug, but no data on long-term side effects in humans are available so far. We thus cannot recommend its nonmedical intake at the present stage.

\section{Christiane Bruns (Cologne, Germany):}

Thank you very much for your presentation. I have 3 questions: How solid is the animal model with respect to production of liver metastases over time? How many animals developed liver metastases after tumor cell injection and how much time was needed to develop liver metastases in this animal model? The second question is, when you analyzed RNA and protein levels of all different factors regarding inflammation, angiogenesis, EMT, and further on, did you handle tumor heterogeneity? Did you pool all liver metastasis of all animals with or without treatment to analyse RNA and the protein levels of the above-mentioned molecular markers?

Furthermore, ITPP - as you said - decreases the binding efficacy of hemoglobin in erythrocytes. So, if you think of a clinical situation, you need to evaluate baseline hemoglobin levels and erythrocyte counts. I haven't seen this. Have you analyzed hemoglobin levels and erythrocyte counts in the animals and have you done this in the starting clinical since this seems to be important for the efficacy of ITPP?

I haven't really understood the underlying biological effect of the drug. It obviously interacts with angiogenesis, inflammation, EMT, and other biological functions. But, would it be possible to really use this in the clinic? Could you please comment on those 3 questions?

\section{Response from Perparim Limani (Zurich, Switzerland):}

Thank you Professor Bruns for your important questions. Regarding the first question, we used an animal model of cecal injection described first over 40 years ago. In this model, over $60 \%$ of the animals develop liver metastases by day 60 after cancer injection, with metastases being first detectable on MRI at day 30. It is thus a fairly robust model of metastatic development.

As for your second question, the study was not set out to consider tumor heterogeneity. We, however, measured parameters in tumors with a diameter of at least $1 \mathrm{~mm}$ to enrich for hypoxic ones (hypoxia usually develops in tumors larger than $200 \mu \mathrm{m}$ ). We analyzed individual tumors, and we targeted tissue between the periphery and the core to analyze hypoxic regions not hampered through necrotic changes. ITPP decreases the oxygen binding affinity of hemoglobin, however has no significant long-term effects on blood parameters in animals (eg, RBC count, hemoglobin, hematocrit etc., see PMID: 21370375). We therefore have not analyzed hematological parameters, which, however, are standard parameters assessed for our patient trial. Thus far, ITPP has not been associated with unwanted side effects (see, eg, http://normoxys.com/clinical-trialresults/) and might even have beneficial effects on cardiovascular/ physical performance of patients (PMID: 19204295) - good conditions for its clinical use.
Finally, the primary working mode of ITPP (ie, via increased oxygen release under low oxygen tension) is the inhibition of tumor hypoxia, which leads to the inactivation of the HIFs and consequently the inhibition of the HIF-dependent hypoxic tumor response (that is angiogenesis, inflammation, EMT, and other biological functions). In our previous publication (PMID: 27489288), we have shown that all survival benefits of ITPP are lost if animals are cotreated with a HIF activator.

\section{Antonio Pinna (Bologna, Italy):}

Thank you Dr. Limani for your interesting study. I have a few brief questions for you. First, you have shown us that overtime the beneficial effect of ITPP is going to decrease. Could you give us an answer for that? Second, you have shown us that the metastatic mass is going to decrease in the model with ITPP and Folfox combined. Could you tell us how did the animals die? And then finally, you correctly stated that the experiment was done to replicate treatment in stage III colorectal cancer. However, I've seen you have already started with the phase- 2 trial in humans where they are stage IV. So, I still think it will probably be interesting to add anti-VEGF in the animal models study combined with ITPP just considering the use for stage IV patients.

What about the long-term efficacy of the ITPP? You have shown us that some molecular beneficial effects of the ITPP can be lost over time. Have you seen a statistical decrease of all the biomarkers that you measured?

\section{Response from Perparim Limani (Zurich, Switzerland):}

Thank you Professor Pinna for your questions. Perhaps I did not present our findings clearly enough, but 1 outstanding feature of ITPP is that its anticancer effects seem to be long-lasting. The reduction in malignancy parameters 6 weeks after treatment was similar to that immediately after treatment - any seeming differences were not significant. In a previous study, we observed an antitumor response stable for at least 4 weeks. How long the ITPP effects may last is unknown, but we assume that once tumor vasculature has been normalized through ITPP (see our study PMID: 27489288), the antihypoxic state may be rather stable. However, we cannot expect the ITPP effects to remain completely stable, since tumors and their biology are likely to change with time.

Regardless of treatment, mice died either of portal compression, caval compression, intestinal congestion, or a combination thereof, indicating that both the growth of primary CRC and secondary liver tumors contributed to mortality. As for the stages, here we used a stage III CRC model, while in our previous study, we demonstrated efficacy for ITPP in a stage IV model (PMID: 27489288, with established liver metastasis). Our clinical trial is on stage IV patients, simply because this is the usual patient population available for first-in-patient studies.

Currently, we would not advise for an ITPP-anti-VEGF combination. ITPP increases oxygenation and thereby causes HIF inhibition followed by VEGF downregulation. Anti-VEGF would additionally reduce VEGF levels likely to the point inhibiting any angiogenesis. This in turn would enhance hypoxia, the primary target of ITPP. It hence seems to make little sense to combine these 2 opposing agents. Of note, ITPP-FOLFOX was much more efficient than anti-VEGF-FOLFOX in our stage IV CRC model.

\section{René Adam (Villejuif, France):}

I really enjoyed your paper. A brief question: The standard treatment today of metastatic colorectal cancer is, as you know, doublets or triplets with targeted therapy. So, I would have been very interested in your animal model to see a group treated with Folfox 
with an antiangiogenic factor like Bevazizumab, because by adding an antiangiogenic effect, we may stipulate that we will have more necrosis, more hypoxia and it will be very interesting to know if ITPP is acting with more activity or not in this setting? What do you think about that?

\section{Response from Perparim Limani (Zurich, Switzerland):}

Thank you Professor Adam for your interesting question. Our mouse CRC model is similar to stage III human disease, for which antiangiogenic agents are not recommended - therefore we did not include anti-VEGF in this study. However, we compared anti-VEGFFOLFOX with ITPP-FOLFOX combinations in our previous study using a "stage IV"-mouse model (PMID: 27489288). There, ITPPFOLFOX led to a markedly improved survival relative to anti-VEGFFOLFOX treatment. With regards to a combination of ITPP with anti-VEGF, we would predict lesser activity for ITPP, because its antihypoxic action would be counteracted by the pro-hypoxic action of anti-VEGF. ITPP alone reduces VEGF levels, while adding antiVEGF may completely block angiogenic activities and thereby promote hypoxia. But you are right, only experimental testing would show whether our prediction is correct. 